\title{
Outcomes of Endovascular Treatment in Acute Ischemic Stroke with Current Malignancy
}

Man-Seok Parka , Bang-Hoon Cho ${ }^{\mathrm{a}}$, Joon-Tae Kim ${ }^{\mathrm{a}}$, Kang-Ho Choi ${ }^{\mathrm{b}}$, Ki-Hyun Cho ${ }^{\mathrm{a}}$, Woong-Yoon ${ }^{\mathrm{c}}$ 'Dept. of Radiology, Chonnam National University Hospital, Gwangju, Korea

\section{Introduction}

The role of endovascular recanalization in the treatment of cancer patients with acute stroke remains elusive. Our study aimed to investigate the clinical and imaging outcomes of endovascular recanalization treatment in patients with acute large vessel occlusion stroke who had active cancer.

\section{Methods}

We reviewed prospectively collected data for ischemic stroke from our stroke registry from January 2011 to September 2016. Acute stroke patients with large artery occlusion in the anterior circulation who had active cancer were identified. Baseline clinical characteristics and postprocedural and long-term clinicoradiological outcomes were evaluated. A good outcome was defined as a 90-day modified Rankin Scale score of 0 to 2. Outcomes were also compared with those of non-malignancy patients who had received endovascular therapy during the same period.

\section{Results}

A total 378 ischemic stroke patients received endovascular treatment, of whom 27 had current malignancy. In patients with current malignancy, a low baseline NIHSS score and male sex were associated with functional independency at 90 days. When comparing with non-malignancy patients, no significant differences in the proportions of patients with symptomatic intracranial hemorrhage and good functional outcome were found in the malignancy patients.
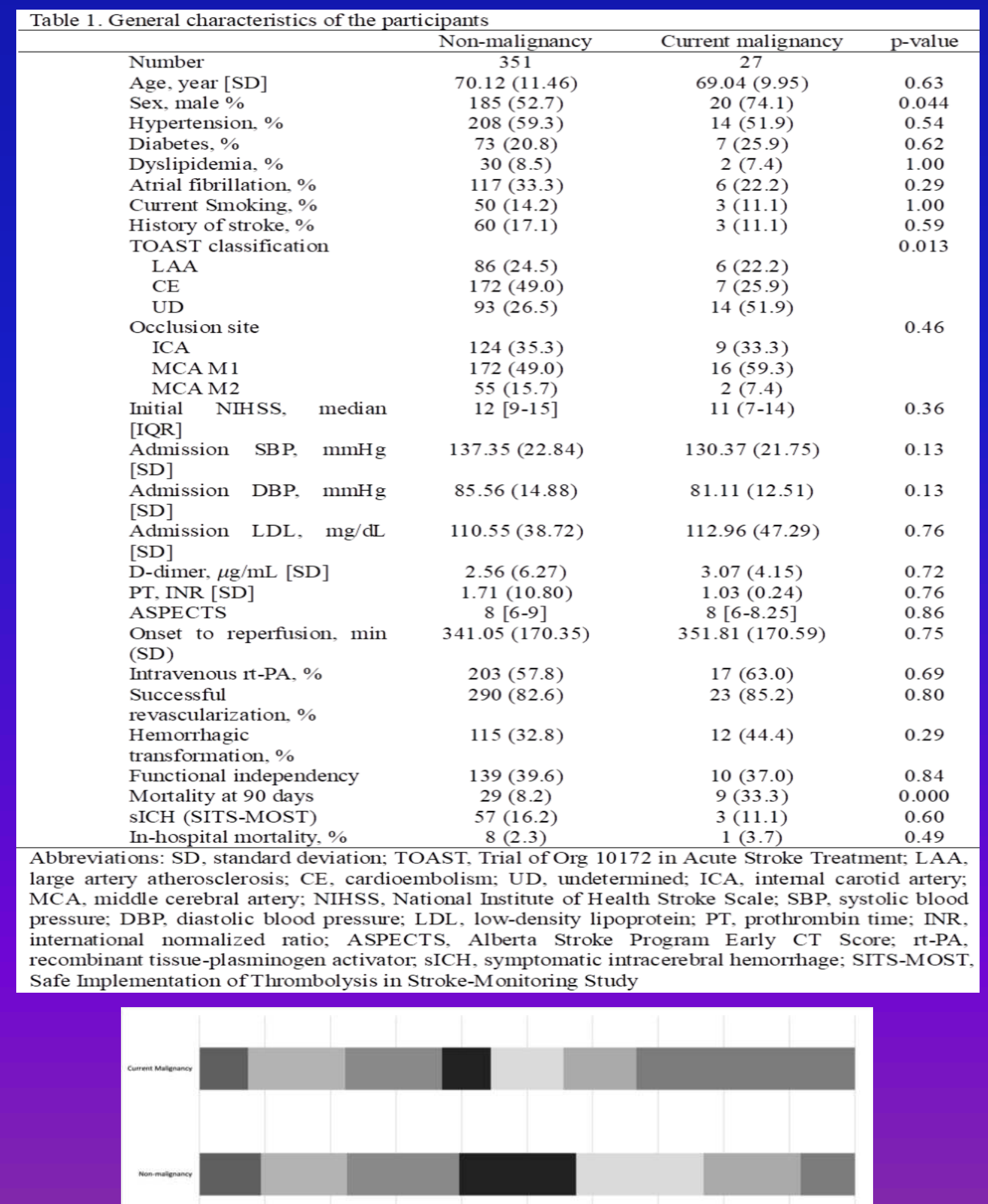

The success rate of recanalization and incidence rate of hemorrhagic transformation were comparable between the two groups $(82.6 \%$ vs $85.2 \%, p=0.80 ; 32.8 \%$ vs $44.4 \%, p=$ 0.29 , respectively). Symptomatic $\mathrm{ICH}$ and in-hospital mortality were not significantly different between the two groups $(16.2 \%$ vs $11.1 \%, p=0.60 ; 2.3 \%$ vs $3.7 \%, p=0.49$, respectively). However, the CM group showed a higher 90day mortality than the non-CM group $(8.2 \%$ vs. $33.3 \%, p<$ 0.001 ). The distribution of 90 -day mRS scores was not significantly different between the two groups $(p=0.07$; Figure).

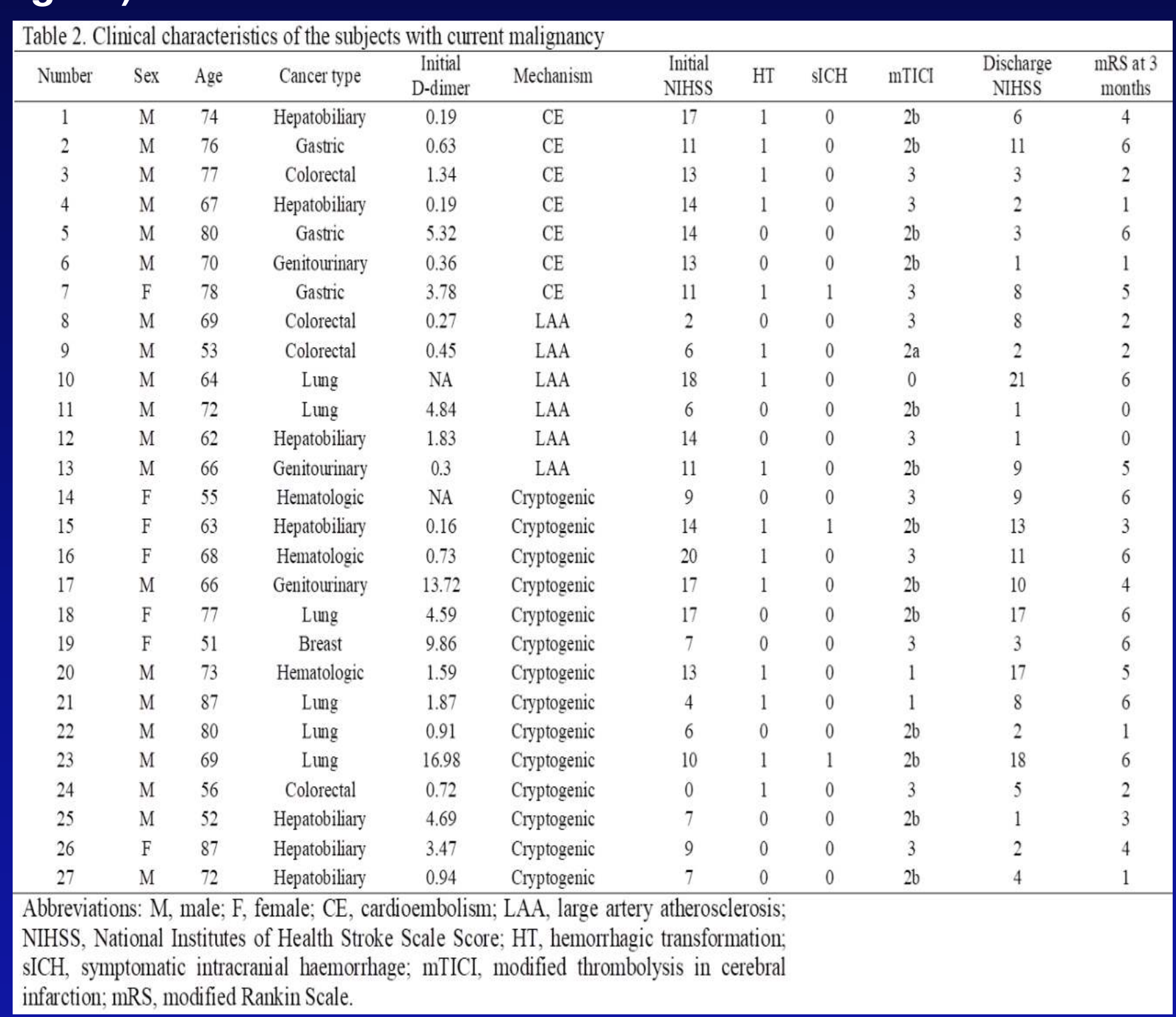

\section{Discussion}

378 AIS patients with large artery occlusion in the anterior circulation received EVT. In the CM group, low baseline NIHSS score was associated with functional independency at 90 days. Functional outcome and radiological findings were not significantly different between the CM and nonCM groups.

The proportions of patients with favorable long-term outcome and hemorrhagic complication in CM group were comparable with those in the past landmark randomized controlled mechanical thrombectomy trials. In the past landmark large clinical trials, the patients with $\mathrm{CM}$ were excluded because the prognosis of cancer patients might be poor and hemorrhagic complications were predicted. Our study showed no significant increase in the incidence of hemorrhagic complications in the CM group after EVT. In the analysis of the CM group, the patients with functional independency at 90 days showed low baseline NIHSS score, more successful reperfusion status, shorter onset to reperfusion time, and low incidence rates of hemorrhagic transformation and sICH. Only low baseline NIHSS score was significantly associated with favorable outcome.

In conclusion, this study suggests that EVT might be feasible for AIS patients with CM. In addition, a low baseline NIHSS score was associated with favorable outcome in this setting. Prospectively designed large clinical studies are necessary. 